\title{
Beitrag zum Problem der heterosynaptischen Facilitation in Aplysia californica *
}

\author{
R. v. Baumgarten and B. JaHan-Parvar \\ Neurophysiologische Abteilung des Mental Health Research Institutes \\ der Universität von Michigan, Ann Arbor/Mich., USA
}

Eingegangen am 17. März 1967

\begin{abstract}
Summary. 1. Heterosynaptic facilitation (H.S.F.) of single neurons in the central nervous system of Aplysia can be repeated virtually indefinitely, provided sufficient time is allowed for recovery between the trials.

2. If the pauses between the pairing trials are too short, a reversible "exhaustion" of the H.S.F. takes place. The test response finally decreases beyond the control level, indicating a, shortage of transmitter.

3. Partial recovery appears when either the priming stimulus or the test stimulus is omitted or given at a lower rate. A complete pause of the stimulation leads within few minutes to full recovery.

4. Continuous priming alone with relatively weak priming shocks but at high rates causes facilitation at first followed by exhaustion of the H.S.F. If paired stimuIation is applied subsequently, the test response increases again. This second facilitation is large but of very short duration. The results indicate that, during a period of priming alone changes occur in some of the test units towards higher responsiveness to subsequent paired stimulation.

5. During repetitive test stimulation alone, the amplitude of the test response diminishes by a decrease of it's unitary fractions without changing configuration. Subsequent paired stimulation augments the size of the unitary E.P.S.P. again, the test response finally approaching the original shape and size.

6. It is suggested that, during H.S.F. both the mobilization as well as the release of transmitter substance are facilitated.
\end{abstract}

Auf der Suche nach Mechanismen, die als Grundlage des Gedächtnisses dienen könnten, sind häufig neurophysiologische Methoden, wie Reizung, Ausschaltung und Ableitung am Säugetiergehirn in Verbindung mit einem Lerntraining angewandt worden [6,19]. Die Resultate solcher Experimente waren aufschlußreich, aber meistens so komplex, daß aus ihnen nur wenig über die eigentlichen Vorgänge in der Feinstruktur geschlossen werden konnte. Wie vielfältig andererseits eine psychologische Lern- und Gedächtnisleistung auch immer sein mag, es erscheint denkbar, daß ihr einfachere Elementarvorgänge auf Zellniveau zugrunde liegen. Die Kompliziertheit kann durch die Vielfalt der neuronalen Verbindungsmöglichkeiten bedingt sein. Es war deshalb besonders zu begrüßen, als

* Mit Unterstützung der Deutschen Forschungsgemeinschaft. 
KANDEL u. TAUO [15-17] anfingen, mit neurophysiologischer Reiz- und intracellulärer Ableitetechnik eine Art klassisch bedingten Reflexes an einzelnen Neuronen im Zentralnervensystem von Aplysia nachzuahmen.

Aplysia, eine große Seewasserschnecke, eignet sich vorzüglich als vereinfachtes Modell zur neurophysiologischen Grundlagenforschung $[1,2,4]$, da ihr Zentralnervensystem aus wenigen Ganglien besteht, die durch lange Connective miteinander in Verbindung stehen. Die zum Teil schon mit unbewaffnetem Auge sichtbaren Riesen-Nervenzellen innerhalb der Ganglien sind erstaunlich konstant in Lage, Farbe, Größe, anatomischen Verbindungen und physiologischen Eigenschaften. Etwa 30 von den Zellen haben deshalb bereits eigene Bezeichnungen oder Namen erhalten $[14,20]$.

Gewisse Informationen können in Aplysia-Nervenzellen offenbar über längere Zeit gespeichert werden [21]. Als Vorstufe des Langzeitgedächtnisses kommen für einige Informationen kurzfristige reversible, wiederholbare Änderungen der Erregbarkeit einzelner Neurone in Frage [8]. Es ist deshalb interessant, nach solchen, einige Minuten überdauernden, aber noch reversiblen Änderungen zu fahnden und ihren Mechanismus zu untersuchen.

Als einer dieser Vorgänge ist die posttetanische Potenzierung bekannt, die seit langem als mögliche Grundlage des Kurzgedächtnisses angesehen wurde $[8,12,18]$. Einen äußerlich ähnlichen, aber vielleicht teilweise doch wesensverschiedenen Mechanismus haben Kandex $u$. TAuc als heterosynaptische Facilitation (im folgenden H.S.F. genannt) an einzelnen Neuronen im Abdominalganglion von Aplysia beschrieben [15]. Es handelt sich hierbei um eine Förderung, welche das intracellulär abgeleitete, erregende postsynaptische Potential (E.P.S.P.) erfährt, wenn der Testreiz an einem afferenten Nerven mit einem „Primingstimulus" genannten, nachfolgenden stärkeren Reiz an einem anderen Nerven nach Art des klassischen bedingten Reflexes zeitlich gepaart wird. Diese Förderung kann in einzelnen Fällen bis zu 20 min nach Ende der Primingreizung anhalten.

Die vorliegende Arbeit handelt vorwiegend von den reversiblen Veränderungen, welche die Testantwort erfährt, wenn der Primingreiz alleine oder gepaart mit dem Testreiz über längere Zeiträume fortgesetzt angewandt wird. In diesen Veränderungen glauben wir deutliche Hinweise dafür zu finden, daß es sich bei der H.S.F. nicht nur um eine heterosynaptische Kontrolle der Ausschüttung, sondern auch der Mobilisierung des Transmitters in den Endformationen der Testneurone handelt. Eine vorläufige Kurzmitteilung wurde in ,Brain Research" [3] veröffentlicht.

Beim Vergleich mit den Bezeichnungen des bedingten Reflexes wäre der Testreiz, der zunächst nur zu einer E.P.S.P. führt, am ehesten mit dem bedingten Reiz, 
und der „Primingstimulus", der meistens ein Aktionspotential hervorruft, mit dem unbedingten Reiz zu vergleichen. Die Schwierigkeit im deutschen Sprachgebrauch liegt nur darin, daß der "Primingreiz" bei gepaarter Reizung nicht zuerst, sondern immer an zweiter Stelle kommt. Mit „Priming" ist nicht die Reihenfolge, sondern eine zusätzliche Maßnahme zur verstärkten Entladung gemeint. Vielleicht wäre ,,verstärkender Reiz" (inforcing stimulus) zweckmäßiger, doch sei in dieser und der folgenden Arbeit die von KANDEL u. TAUC gewählte Bezeichnung beibehalten, um den Vergleich mit den grundlegenden Arbeiten dieser Autoren, auf denen sich vorliegende Untersuchung aufbaut, zu erleichtern.

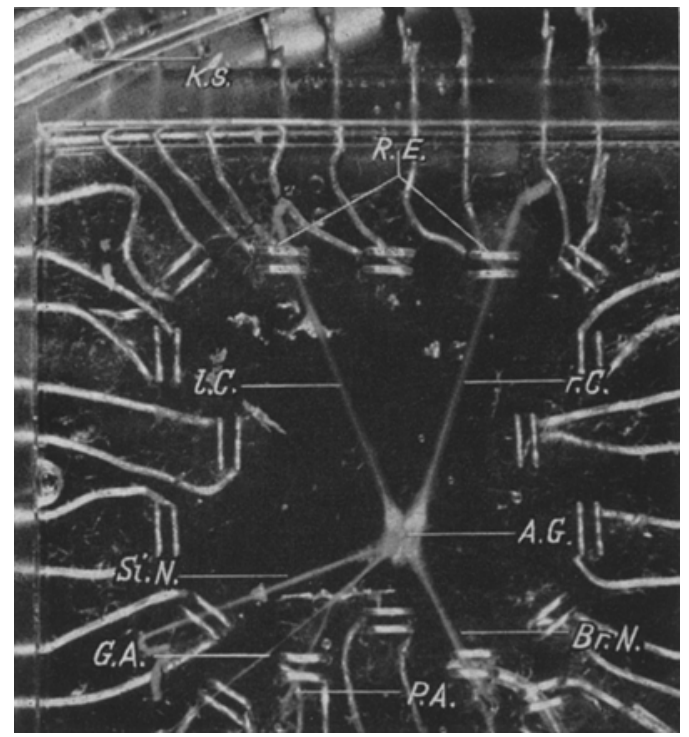

Abb. 1. Ableitlammer mit Abdominalganglion. A.G. Abdominalganglion, Br.N. Branchialnerv; G.A. Genitalast; K.S. Kühlschlauch; l.C. linkes Connectiv; P.A. Perikardialast; r.C. rechtes Connectiv; R.E. Reizelektroden; Si.N. Siphonnerv. Die ganze Ableitkammer ist mit Seewasser gefüllt. Der Kühlschlauch liegt im äußeren Teil der Kammer und ist mit gekühltem Wasser durchströmt. Die Reizelektroden liegen im inneren Teil und ragen nur mit ihrer blanken Oberfläche aus der gummiartigen Einbettungsmasse (Silastik) hervor. Die sechs Nerven des Ganglions sind jeweils auf Reizelektroden gelegt und mit feinsten Insektennadeln auf der Unterlage befestigt worden

\section{Methodik}

Präparation. Die Experimente wurden an über 60 Aplysien (Aplysia californica) vorgenommen. Die Tiere kamen frisch per Luftfracht in gekühlten Behältern aus Californien und waren in gutem Zustand. Sie wurden bis zum Gebrauch in einem gekühlten Seewasseraquarium mit biologischer Filteranlage (Instant ocean, culture system, Fa. Aquarium Systems, Inc., Wicklife, Ohio) gehalten. Das Seewasser wurde aus ,instant ocean" derselben Firma in einfacher Weise künstlich zubereitet. Die Ganglien wurden in der üblichen Weise rasch aus der Schnecke herauspräpariert und in die Ableitkammer gebracht. Während wir anfangs nur das Abdominalganglion herauspräparierten, war es später im Hinblick auf simultane Ableitungen mehrerer 
Ganglien manchmal zweckmäßig, das ganze Zentralnervensystem herauszupräparieren, und mit allen Ganglien in natürlichem Zusammenhang zu verwenden.

Ableitkammer. Die Ableitkammer bestand aus einer $10 \times \mathbf{1 0} \mathrm{cm}$ großen rechteckigen Schale, deren Boden mit Silastic, einer gummiartigen, durchsichtigen Masse, ausgegossen war (Abb.1). Die Ganglien wurden darauf in annähernd natürlicher gegenseitiger Positition mit Insektennadeln befestigt. Die Ableitkammer wurde mit $120 \mathrm{~cm}^{3}$ künstlichem Seewasser gefüllt, das öfters erneuert wurde. Die Temperatur wurde mit einer Kühlschlange konstant auf $15^{\circ} \mathrm{C}$ gehalten. In die Silasticmasse waren 20 bipolare Reizelektroden aus Platindraht mit blanker Oberfläche eingelassen, außerdem zwei spiralförmige indifferente Elektroden aus Platin. Die Reizelektroden waren mit einem Schaltkasten verbunden, der es erlaubte, jede der Reizelektroden wahlweise auf eines von zwei Rechteckreizgeräten bzw. deren Hochfrequenzisolierungsgeräte zu schalten. Alle freipräparierten Nerven und Connective des Z.N.S. wurden auf je eine der Reizelektroden gelegt, so daß es möglich war, durch Probereizungen die wirksamsten Kombinationen von Test- und Primingreiz zu ermitteln.

Reizart. Als Testreiz wurden einzelne Rechteckreize von 2 msec Dauer und leicht überschwelliger Stärke in Abständen von 5-20 sec angewandt. Vor Beginn der Primingreizung wurde ein ,steady state" der Testantwort bei der gewünschten Reizfrequenz abgewartet. Der Primingreiz bestand meistens aus einer 1 sec langen Serie von vier submaximalen Rechteckreizen, die bei gepaarter Reizung $350 \mathrm{msec}$ nach dem Testreiz begannen. In bestimmten Fällen wurde auch mit Erfolg ein einzelner Rechteckreiz als Primingreiz verwendet. Da die Testantwort bei frequenter Testreizung zunächst abnimmt [16], wurde als Kontrolle die Höhe des Test-E.P.S.P.s nach Erreichen eines ,steady state" gewertet.

Ableit- und Registriertechnik. Es wurden mit 2,5 mol KCl-Lösung gefüllte Capillarelektroden mit einem Spitzenwiderstand zwischen 3 und 5 Megohm benutzt, die mit Hilfe eines Prior-Manipulators unter mikroskopischer Kontrolle in die Zelle eingeführt wurden, Eröffnung der Bindegewebshülle war meistens nicht notwendig. Die Potentiale wurden über einen Bak unity gain-Verstärker einem TektronixKathodenstrahloscilloskop zugeleitet und dort, gleichstromverstärkt (oder wenn notwendig, kondensator-widerstand-gekoppelt) im reizsynchronisierten Einzelkipp zur Darstellung gebracht. Eine mit dem Reizgerät und dem Kathodenstrahlkipp gekoppelte Grass-Kamera mit stufenweisem reizsynchronisierten Filmvorschub diente zur Registrierung, in einigen Fällen auch eine Polaroidkamera.

Eine Brückenschaltung üblicher Bauart ermöglichte künstliche Polarisation der Zellmembran, intracelluläre Rechteckreizung und Abschätzung des Membranwiderstandes über die Ableitmikroelektrode.

\section{Ergebnisse \\ 1. Wiederholbarkeit der H.S.F.}

Unter den Voraussetzungen, daß der Primingreiz nicht exzessiv hoch gewählt, und vor allem eine ausreichende Pause von etwa $15 \mathrm{~min}$ zwischen den gepaarten Reizungen gegeben wurde, war es möglich, die H.S.F. nahezu beliebig oft zu wiederholen. Wenn die Mikroelektrode nicht aus der Zelle rutschte oder aus anderen Gründen die ganze Präparation verdarb, war es möglich, die H.S.F. in über 30 Fällen ausnahmslos öfter als 20 mal, in einem Falle über 60 mal, mit gutem Erfolg zu wiederholen (Abb.2). Die H.S.F. war in allen geprüften Neuronen, die überhaupt 
A

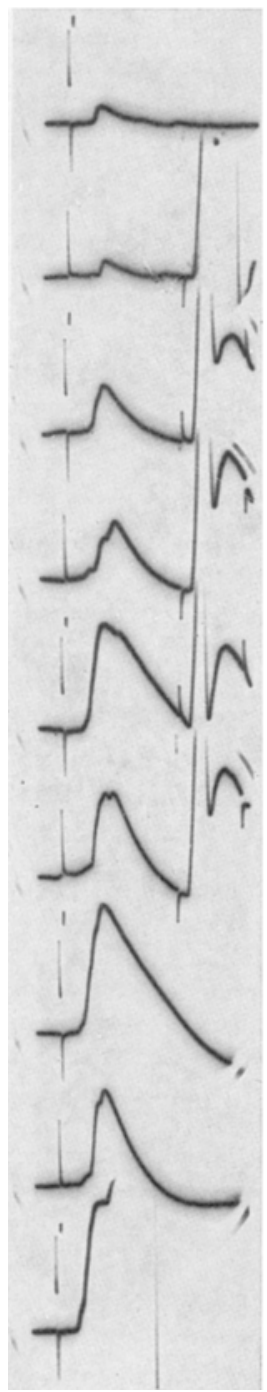

B

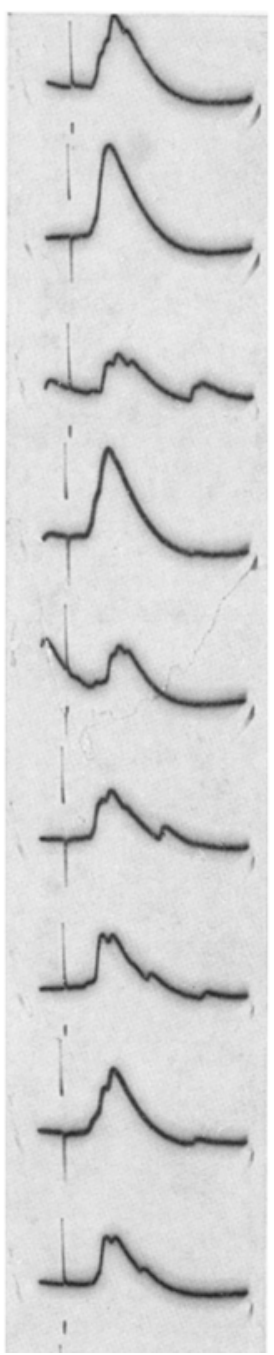

C

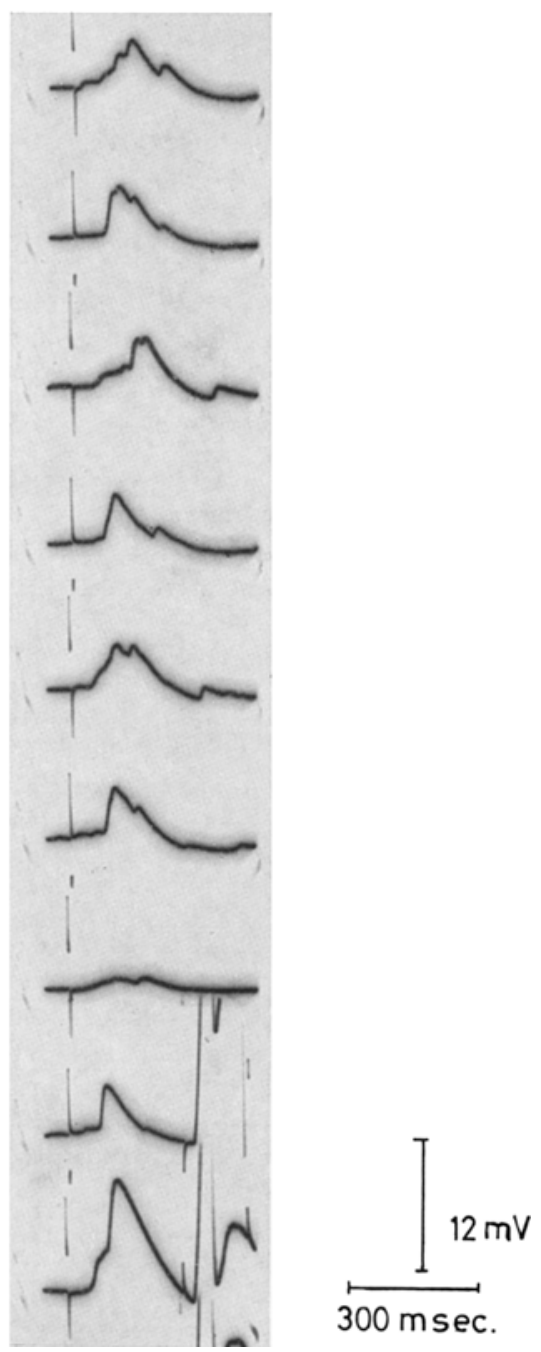

Abb.2. Wiederholbarkeit der heterosynaptischen Facilitation. Testnerv: Genitalnerv, Primingnerv: Siphonnerv. Die oberste Linie in A zeigt die Kontrolle des TestE.P.S.P.'s nach Erreichen der steady state. Diesen Kontrollen sind bereits 58 H.S.F.s und eine anschließende Reizpause von $30 \mathrm{~min}$ vorausgegangen. Es folgt fünfmalige Paarung mit dem Primingreiz. Es tritt eine sehr deutliche H.S.F. ein, die in der letzten Linie von A sogar zum Spike führt. Die kontinuierlichen Abbildungen in B und $\mathrm{C}$ zeigen ein langsames Wiederabklingen der H.S.F., und in den letzten beiden Linien von $\mathrm{C}$ tritt durch gepaarte Reizung erneute H.S.F. ein. Der Abstand zwischen den Testreizungen beträgt 5 sec 
H.S.F. zeigten, auch wiederholbar. Nur bei supramaximalen Primingreizen trat gelegentlich ein irreversibler Ausfall der H.S.F. ein.

\section{Erschöpfung durch gepaarte Reizung}

Wurden die Pausen zwischen den Paarungsserien relativ kurz gewählt (in der Größenordnung von 1-2 min) oder kontinuierliches Priming angewandt, so trat in allen Fallen schlieBlich eine Abnahme der H.S.F. bis zur Erschöpfung ein (Abb. 3). Nach einer nachfolgenden Ruhepause zeigt sich der alte Reizerfolg.

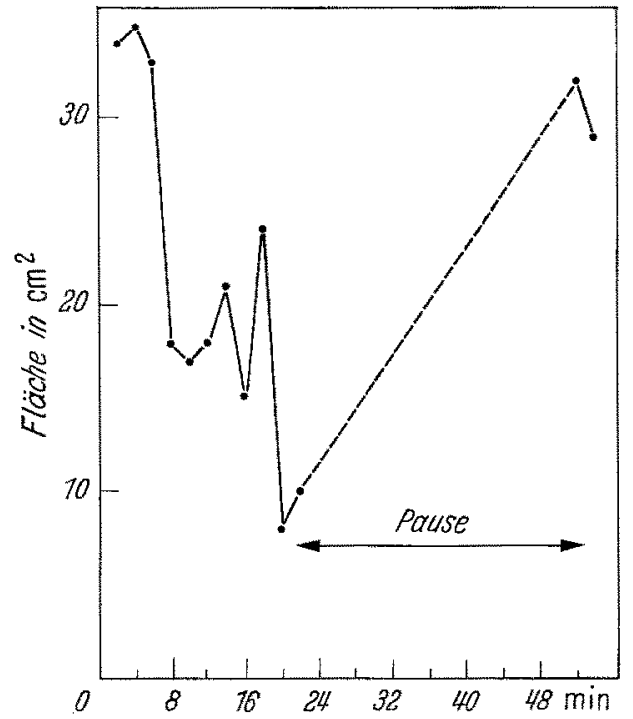

Abb.3. Erschöpfung und Erholung der H.S.F. in Abhängigkeit von der Länge der Reizpausen. Jede Primingserie bestand aus fünf Gruppen von je vier Einzelrechteckreizen mit einem Gruppenabstand von je 5 sec. Die Kurve wurde aus dem Versuch der Abb.1 gewonnen. Die Ordinate eines jeden Kurvenpunktes entspricht der Fläche der Amplitudenkurve aller Testantworten von Beginn zu Beginn zweier Primingserien und somit der ungefähren Transmitterausschüttung. Zu Beginn der Kurve wurde bei der 48. gepaarten Reizung von einer selteneren auf eine relativ häufigere Reizung von je einer Primingserie alle 2 min übergegangen. Die Größe der H.S.F. nimmt daraufhin erheblich ab. Nach einer Reizpause von 30 min tritt eine Erholung bis zum ungefähren Ausgangswert ein. Die Ableitung der letzten beiden Kurvenpunkte ist in Abb.2 dargestellt

Bei diesen Versuchen sank in der Erschöpfungsphase die Testantwort gewöhnlich bis unter den Kontrollwert vor der Primingreizung und erholte sich dann wieder in der Reizpause bis auf diesen Wert.

\section{Erschöpfung durch den Primingreiz alleine}

Kontinuierlich fortgesetzte Reizung mit maximalen Primingreizen im Abstand von $10-20 \mathrm{sec}$ führte zunächst zu einer Steigerung des Test- 
reizes, die mit der unspezifischen Form der H.S.F. (KANDEL u. TAUC) verglichen werden kann. Wurde die Primingreizung aber weiter forgesetzt, so nahm die Amplitude des Testreizerfolges wieder ab und unterschritt schließlich die Höhe des Kontrollwertes. Eine bis unter den Kontrollwert erschöpfte Testeinheit war auch nicht mehr in der Lage, H.S.F. zu entwickeln.

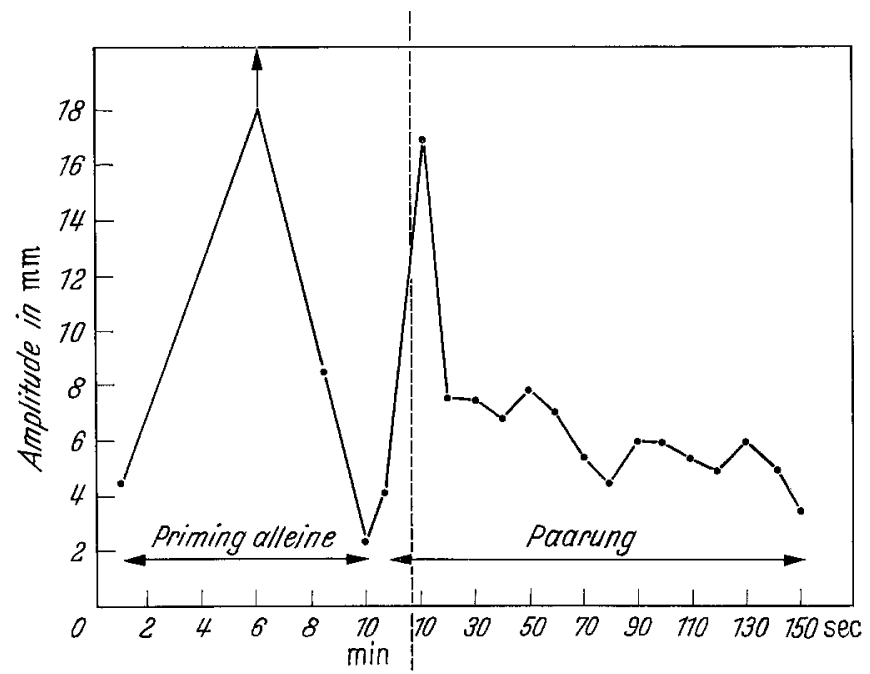

Abb.4. Zeitverlauf der H.S.F. nach Erschöptung durch Priming alleine mit nachfolgender gepaarter Reizung. Der Kurve liegen die Ableitungen der Abb.5 zugrunde. Primingnerv: Siphonnerv, Testnerv: Genitalnerv. Zu Beginn der Kurve führt Priming alleine zu einer unspezifischen H.S.F. mit Spikeproduktion. Fortgesetzte Primingreizung mit einer Reizserie alle 5 sec führt nach 10 min zur Erschöpfung der Testantwort. Wie bei der nun gedehnten Zeitachse zu sehen ist, führt aber weiter fortgesetzte Paarung zu erneuter Erschöpfung

Wurde der Primingreiz auf einen submaximalen Wert eingestellt, so trat offenbar nicht bei allen Testeinheiten Erschöpfung ein. Zunächst nahm der Testreizerfolg zwar ebenfalls zu, um dann abzusinken und unter den Kontrollwert zu fallen. Wurde dann aber sofort anschließend gepaarte Reizung angewandt, so resultierte ein erneuter Anstieg des Testerfolges (Abb.4). Die durch Paarung, nach vorhergehendem Priming alleine erzielte Steigerung des Testerfolges, unterschied sich von der H.S.F. nach Paarung an einer frischen oder ausgeruhten Präparation außerdem durch ihre Kurzlebigkeit (Abb.5). Offensichtlich wurden bei submaximalem Priming einige Testeinheiten nicht entladen und erschöpft, sondern im Gegenteil so beeinflußt, oder ,,vorbereitet", daß eine nachfolgende Paarung einen stärkeren Reizerfolg zeigte. Abb.5 zeigt 
Ausschnitte der Originalableitungen, welche der Kurve von Abb. 4 zugrunde liegen. Ähnliche Beobachtungen wurden in weiteren fünf Fällen gemacht.

Fortgesetzte unausgesetzte Testreizung alleine mit einer Frequenz von über zwei pro Sekunde konnte ebenfalls zu einer Art Erschöpfung führen in welcher eine H.S.F. dann nicht mehr auslösbar war.

1.

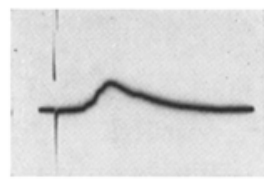

2.

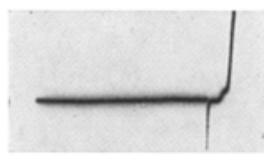

3.

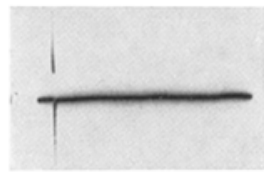

4.

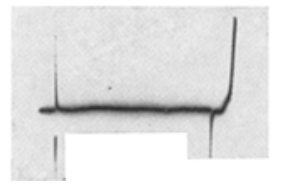

5.

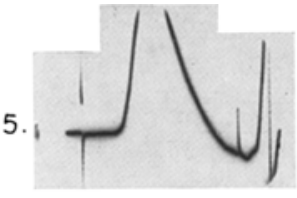

6.
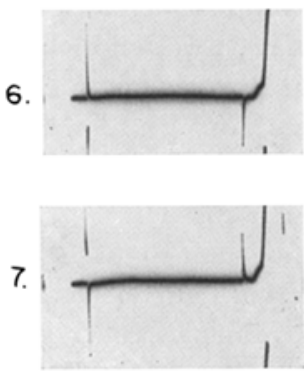

8.

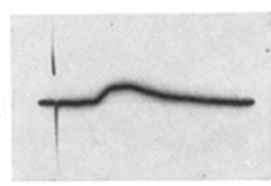

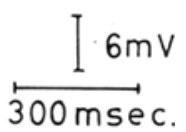

Abb.5. Wirkung lange fortgesetzter Primingreizung mit nachfolgender gepaarter Reizung. Primingnerv: Siphonnerv, Testnerv: Genitalnerv. 1 Kontrolle, 2 Primingreizung alleine, 3 Erschöpfung der Testantwort nach $171 / 2$ min Priming alleine. Eine Priminggruppe von vier Rechteckreizen mit einer Frequenz von 6/seo erfolgte alle 5 sec. 4 Einmalige gepaarte Reizung, 5, 6 und 7 sind kontinuierliche Ableitungen. Bei 5 ist eine außerordentlich starke H.S.F. mit Spike durch die vorangegangene gepaarte Reizung eingetreten. Die nachfolgenden Testreize bleiben unbeantwortet.

8 Nach einer kurzen Reizpause stellt sich die Testantwort wieder ein

Erholung im Sinne einer Wiederauslösbarkeit der H.S.F. war möglich durch zeitweises Weglassen der gepaarten Reizung, des Primingreizes, oder des exzessiv oft angewandten Testreizes. Am wirksamsten war eine totale Reizpause von über 15 min Dauer.

Die Beobachtung, daß wiederholte Anwendung der submaximalen oder maximalen Primingstimulation zum Ausfall der (unspezifischen) H.S.F. führt, wirft die Frage auf, ob es sich dabei um eine Erschöpfung der Primingeinheiten, der Testeinheiten, oder von beiden handelt. Zur 
Prüfung dieses Problems wandten wir fortgesetztes Priming ein und desselben Nerven bis zur Erschöpfung der H.S.F. an und wechselten dann die Primingreizung schnell auf einen anderen,,frischen" Nerven über. In einem Falle wurde auch dadurch die Erschöpfung der Testantwort nicht behoben, in einem anderen Falle zeigte sich, daß zunächst eine partielle Erholung und Restaurierung der H.S.F. zu beobachten war (Abb.6). Da es sich dabei aber nach dem Umschalten um zusätzliche heterosynaptische Förderung frischer, bisher nicht entladener (und erschöpfter) Testeinheiten gehandelt haben kann, schalteten wir nach weiteren 17 min fortgesetzten Primings, nachdem die Testantwort erneut erschöpft war, wieder die Primingreizung auf den ersten Nerven zurück. Die Einheiten dieses Primingnerven hatten nun ausreichend Zeit, sich zu erholen, was aus Kontrollversuchen mit gleich langen oder gar kürzeren Pausen hervorgeht (Abb.6, Teil 11 und 12). Dennoch fiel die H.S.F. auch nach dem Umschalten weiterhin aus, was für eine Erschöpfung der Testeinheiten spricht.

\section{Amplitudenänderung des Testreizerfolges als Folge von Größenänderungen einzelner E.P.S.P.s}

Da die Vergrößerung der Testantwort bei der H.S.F. sowohl an der Summation einer vermehrten Anzahl von E.P.S.P.s, als auch an einer Vergrößerung einzelner E.P.S.P.s liegen kann, wurde bei Anwendung eines hohen Verstärkungsgrades die Konfiguration der Test-E.P.S.P.s vor und nach H.S.F. untersucht und mit der spontanen Amplitudenverminderung bei Übergang zu frequenter Testreizung verglichen (Abb.7). Wenn nach einer längeren Erholungspause der Testreiz gegeben wurde, so resultierte zunächst ein Spike oder zumindest ein besonders hohes E.P.S.P., welches sich dann im Laufe der nachfolgenden Testreizungen bis auf einen von der Reizfrequenz abhängigen „steady state“ verminderte. Die Konfiguration bzw. Form der einzelnen komplexen E.P.S.P.s wurde dabei verhältnismäßig gut gewahrt, was gegen eine Änderung in der Zahl der beteiligten Synapsen spricht. Wie man sieht, beruhte die Verkleinerung vielmehr auf einer, wahrscheinlich auf Transmitterverknappung beruhenden, Amplitudenverminderung der einzelnen Stufen, die offenbar ,,unitary “ E.P.S.P.s darstellen. H.S.F. führt in spiegelbildlicher Weise umgekehrt zu einer Vergrößerung der einzelnen Stufen, und somit zu einem größeren Gesamt-E.P.S.P.

Abb. 8 zeigt eine fortlaufende Ableitung der spontanen E.P.S.P.s vor, während und nach der Primingreizung. Das kleine Potential in a ist seiner Form und seinem, ,alles oder nichts"-Charakter nach vermutlich ein „,unitary“ E.P.S.P. Die Formgleichheit dieses spontanen Potentials mit denen in den folgenden Abbildungsteilen dargestellten, macht es wahrscheinlich, daß es sich um ein und dasselbe synaptische Potential handelt, welches durch die Primingreizung an Amplitude wesentlich zugenommen 


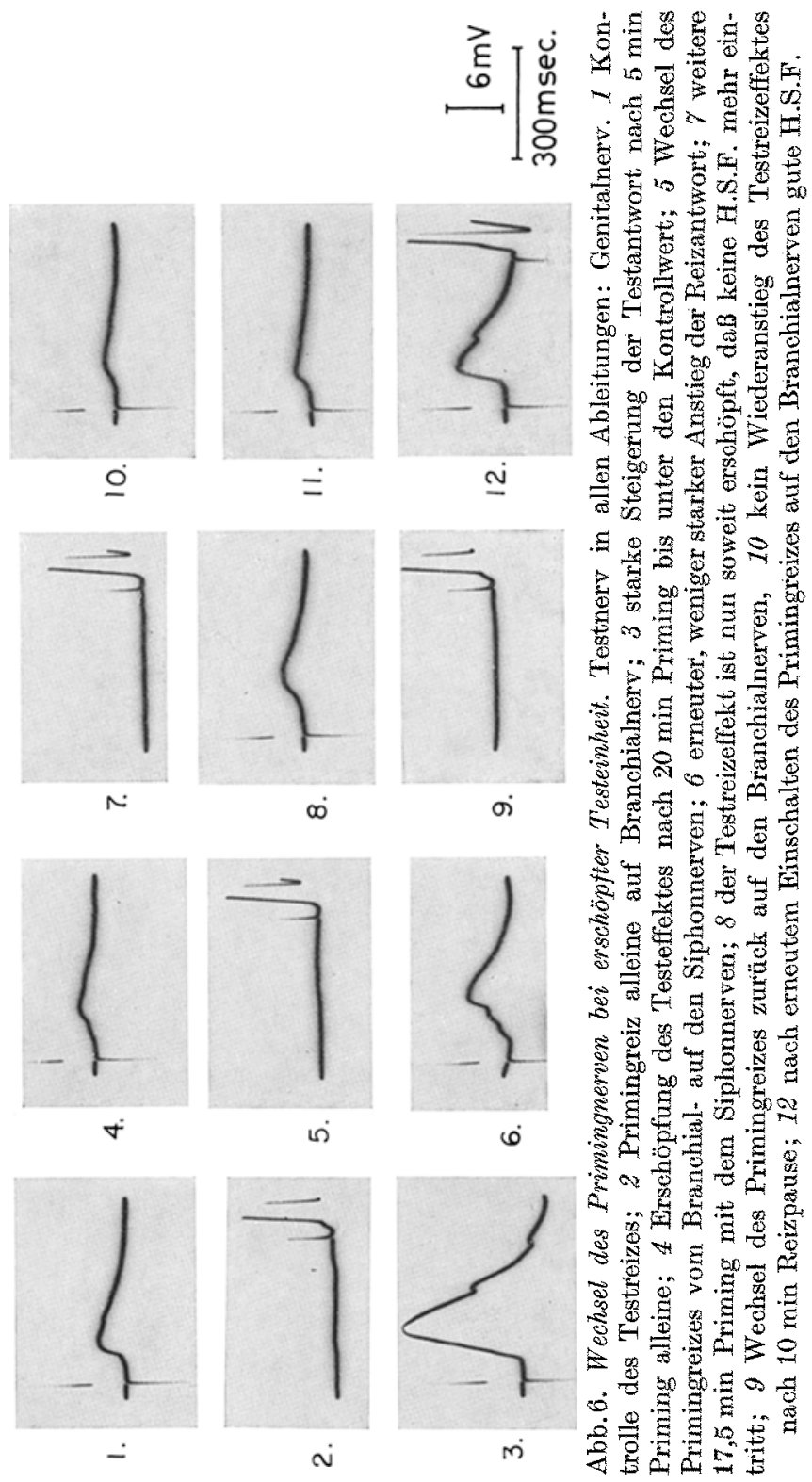

hat und dann langsam wieder auf seinen Kontrollwert zurückgeht. Eine wesentliche Frequenzsteigerung durch Aktivierung von Zwischenneuronen war nur unmittelbar nach der Primingreizung zu beobachten. $\mathrm{Ob}$ die sehr kleinen zusätzlichen Exkursionen der Grundlinie über die 


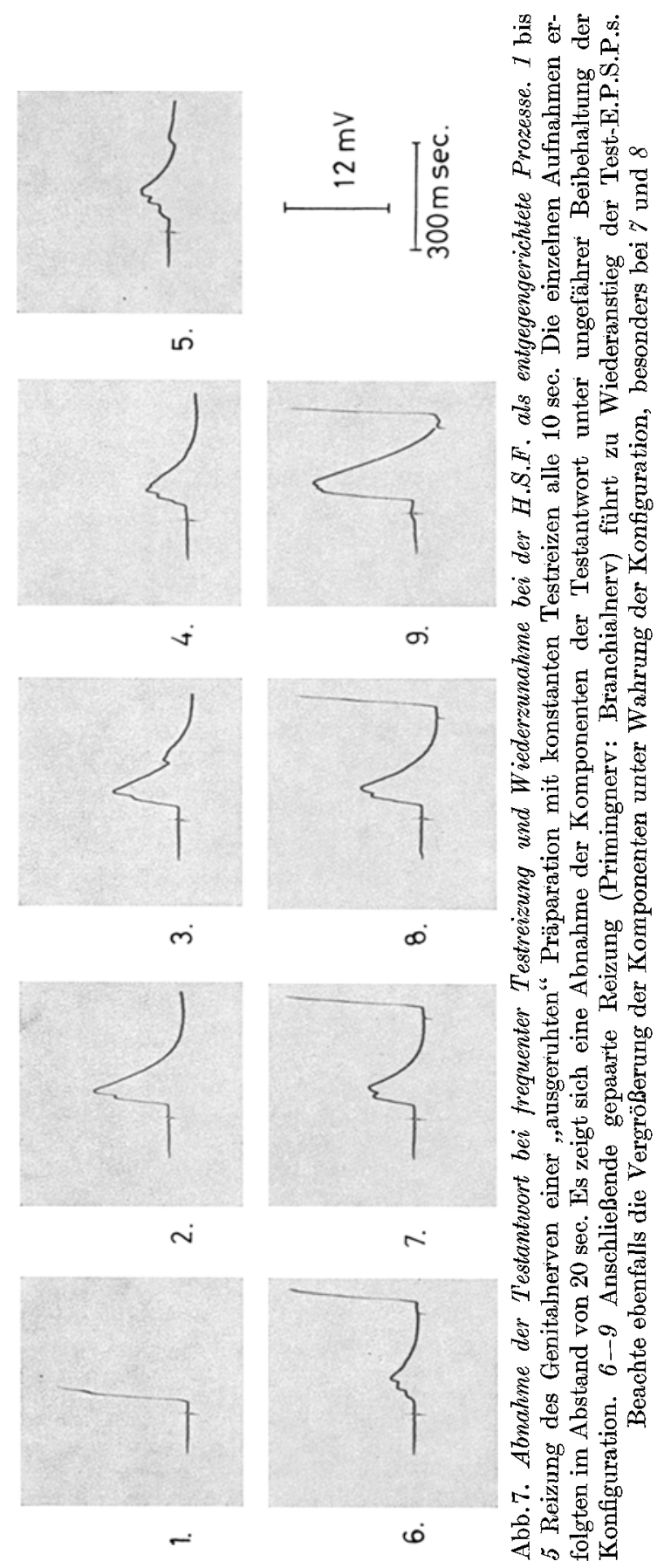



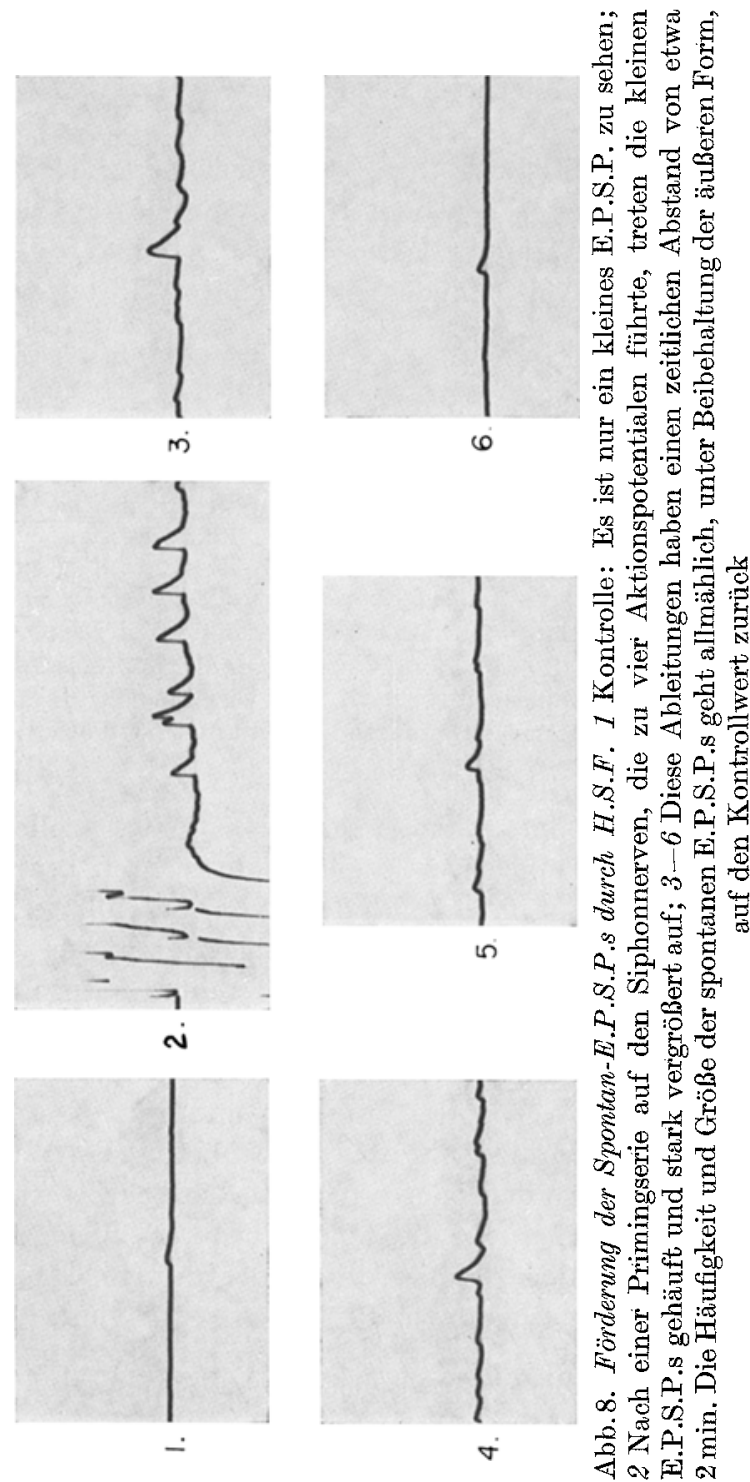

Schwelle der Sichtbarkeit gehobene, weit entfernte synaptische Potentiale darstellen, Miniaturpotentiale eines in Quanten austretenden Transmitters sind, oder rekrutierte Zwischenneurone anzeigen, läßt sich nicht sicher beurteilen.

\section{Allgemeine Beobachtungen}

Die Größe der H.S.F. schwankte beträchtlich in verschiedenen Präparationen, auch für dieselbe Zellart. Im Abdominalganglion hatte der 
Siphonnerv auch bei uns durchschnittlich den besten Primingerfolg [16]. Als Testnerv bot das linke Connectiv eine besonders ausgiebige H.S.F., zeigte aber andererseits oft eine fortschreitende Adaptation an den Testreiz, so daß der Reizerfolg auf lange Dauer nachließ. Beim rechten Connectiv war der Reizerfolg konstanter, doch mußte der Testreiz klein gehalten werden, wenn mit der Riesenzelle gearbeitet wurde, damit deren im rechten Connectiv verlaufendes Axon nicht antidrom erregt wurde.

1.

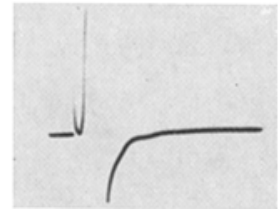

2.

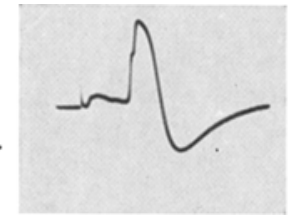

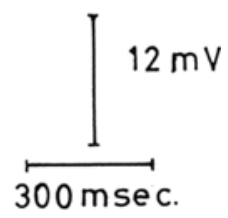

Abb.9. Antidrome Entladungen in der R.O.Q.-Riesenzelle nach Reizung des Branchialnerven. 1 Antidromes Aktionspotential mit kurzer Latenz der R.O.Q.-Riesenzelle nach relativ starker Reizung. Der Befund spricht dafür, daß eine Axonkollaterale der Riesenzelle im Branchialnerven verläuft. 2 Bei verminderter Reizstärke tritt ein orthodrom übertragenes komplexes postsynaptisches Potential auf

Antidrome Aktionspotentiale in der R.O.Q.-Riesenzelle ließen sich sowohl vom rechten Connectiv als auch vom rechten Branchialnerven auslösen. Offenbar sendet diese Zelle efferente Axon-Kollateralen in beide Nerven (STrumwasser [21]). Die notwendige Reizstärke zur Erregung dieser Fasern war größer als für viele der afferenten Fasern (Abb.9).

Das herauspräparierte und in der Ableitkammer ,aufgespießte“" Z.N.S. der Aplysia überlebte bemerkenswert lange. Es war ohne weiteres möglich, den Versuch abends abzubrechen und am anderen Tage, am selben Ganglion, wieder aufzunehmen. Hierbei konnte die Mikroelektrode in der Zelle belassen werden oder erneut in dieselbe Zelle eingestochen werden. In einem Falle wurde die Ableitung bei häufigem Wechsel des Seewassers und Kühlung der Ableitkammer auf $15^{\circ} \mathrm{C}$ ohne sonstige weitere Kultivierungsmaßnahmen auf 6 Tage ausgedehnt.

\section{Besprechung der Ergebnisse}

Wenn die unter Laboratoriumsbedingungen erzielte heterosynaptische Facilitation ein Modell eines physiologischen Vorganges am Ganztier sein soll, so ist unter anderem zu fordern, daß sie beliebig oft wiederholbar ist. Dieses schien nach den ersten Erfahrungen Kandels u. Taucs [16], die eine H.S.F. nur sechsmal hintereinander auslösen konnten, nicht der Fall zu sein. In vorliegender Arbeit konnte aber ergänzend gezeigt werden, da $\beta$ bei Verwendung frischer Tiere, submaximalem Primingreiz und ausreichender Erholungszeit zwischen den Versuchen die Wiederholbarkeit praktisch nicht begrenzt ist. 


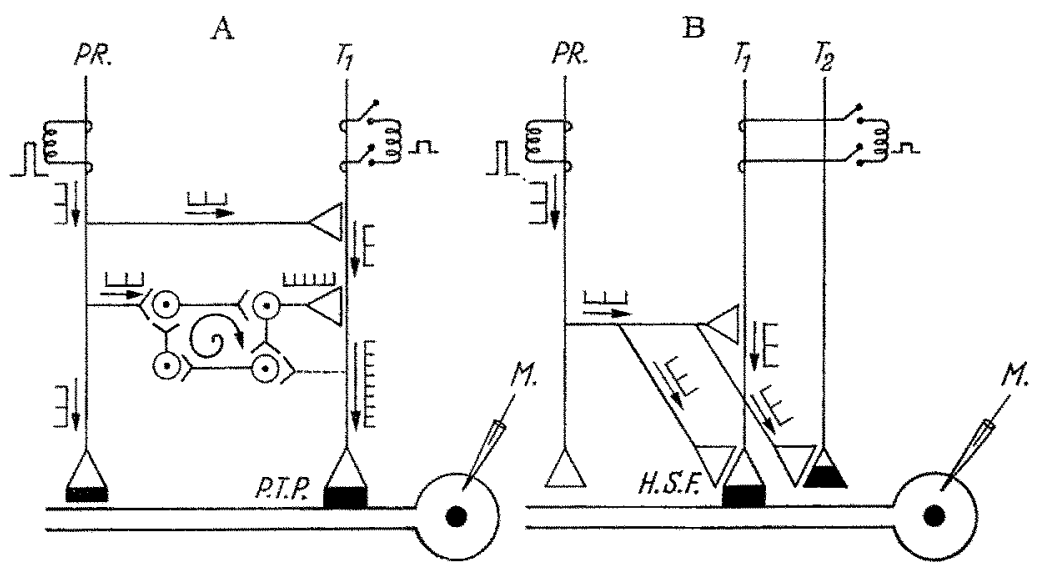

Abb. 10. Hypothetische Schemata zur Interpretation der Befunde. M Mikroelektrode, Pr Primingneuron, P.T.P. Posttetanische Potenzierung, $T_{1}$ und $T_{2}$ Testneurone. Die Pfeile mit den kammförmigen Symbolen sollen die FluBrichtung und die relative Häufigkeit der Aktionspotentiale darstellen. In beiden Fällen wurde Reizung durch Priming alleine angenommen. $\mathrm{Ob}$ es sich, wie aus Einfach heitsgründen angenommen, um multiple Kollateralen jeder einzelnen Primingeinheit handelt oder um verschiedene Endigungen mehrerer Primingneurone, spielt in diesern Zusammenhang keine Rolle

A. Posttetanische Potenzierung. Posttetanische Potenzierung der Testendformation durch direkte fördernde Verbindungen des Primingneurons mit dem Testneuron, außerdem durch reverberierende Entladungen im Netz der Zwischenneurone. Im Falle, daß die Testunit ihrerseits Verbindungen zu gemeinsamen Interneuronen mit der Primingseite hat (gestrichelt dargestellt), müßte außerdem mit ,subliminal fringe"-Förderung gereehnet werden

B. Präsynaptische Beeinflussung der Testneurone. Die Endformationen beider Testneurone haben präsynaptische Verbindungen von der Primingseite her, welche die Transmitterbereitstellung fördern. Testneuron I hat anßerdem eine direkt fördernde Afferenz und wird infolgedessen vom Primingreiz alleine schon entladen und entleert. Testneuron 2 hat keine direkt födernde Verbindung und kann infolgedessen den bereitgestellten Transmitter speichern, bis eine nachfolgende gepaarte Reizung zur Ausschüttung führt

Hinsichtlich des Mechanismus der H.S.F. bestehen, da Veränderungen an der postsynaptischen, abgeleiteten Membran ausgeschlossen werden können [16,17], grundsätzlich noch mindestens drei Möglichkeiten (Abb. 10):

1. Es handelt sich um eine vermehrte Summierung von EinzelE.P.S.P.s durch allgemeine Aktivitätsteigerungen im Netz der Zwischenneurone. Diese führt zur Ausweitung des unterschwelligen Erregungssaumes (subliminal fringe) an den mit der abgeleiteten Zelle synaptisch verbundenen Neuronen. Hierdurch werden nach vorausgehender Primingreizung mehr Neurone pro Testreiz entladen als vorher. 
2. H.S.F. beruht auf einer posttetanischen Potenzierung der Transmitterausschüttung durch frequente Spike-Entladungen der Endigung des Testneurons. Solche Entladungen können sowohl durch direkte fördernde Verbindungen von der Primingseite her, als auch durch aktivierte reverberierende Erregungskreise verursacht worden sein.

3. Die Transmitterbereitstellung, und damit die Menge der Transmitterausschüttung des Testneurons wird durch Nervenendigungen der Primingneurone an den Testneuronen (vielleicht präsynaptischer Art) kontrolliert, welche an diesen zwar kein Aktionspotential, aber eine graduelle Antwort hervorrufen (vielleicht auch chemische Stoffe übertreten lassen).

Kandec u. Tauc gaben dieser dritten Möglichkeit den Vorzug. Wir halten aufgrund unserer Beobachtungen die Realität aller drei Mechanismen für möglich, die zu verschiedenen Zeitabschnitten gemeinsam zur Amplitude des komplexen Test-E.P.S.P.s bei der H.S.F beitragen.

Die allgemeine Aktivitätszunahme läßt sich leicht bei fortlaufender Ableitung nach Primingreizung an der vermehrten Anzahl der einzelnen Spontan-E.P.S.P.s beobachten (Abb.8). Die beteiligten Interneurone müßten allerdings Verbindungen zu den Testeinheiten haben, um H.S.F.wirksam zu sein. Diese Verbindungen sind unbewiesen, aber ihr Vorhandensein im Neuropil erscheint durchaus möglich.

Posttetanische Potenzierung ist bei dem Teil der Testneurone zu erwarten, die von der Primingseite selbst, und außerdem vielleicht auch von aktivierten $Z$ wischenneuronen mehrfach entladen werden. Daß nach Reizung des Primingnerven alleine ein Teil der Testneurone entladen werden, geht daraus mit großer Wahrscheinlichkeit hervor, daß wiederholtes Priming alleine zur Erschöpfung der H.S.F. und sogar der Testantwort bis unter den Kontrollwert führen kann.

Interneuronenaktivierung und posttetanische Potenzierung kommen mehr für den frühen Teil der H.S.F in Frage, da ihr Zeitverlauf begrenzt ist (siehe auch [17]). Auch nach unserer Erfahrung bleiben einige Beobachtungen bestehen, die nicht mit diesen beiden „klassischen“ Mechanismen erklärt werden können und deshalb besser in die dritte Gruppe, nämlich der heterosynaptischen Kontrolle der Transmitterbereitstellung und -ausschüttung passen. Wenn diese im folgenden abgehandelten Mechanismen auch nicht notwendigerweise den einzigen Faktor bei der H.S.F. darstellen, so scheinen sie uns aufgrund ihrer Neuartigkeit, und weil sie Beziehungen zu den spezifischen Lernvorgängen haben könnten [16], besonders darstellenswert.

Die „Summationstheorie“" (siehe oben) erklärt nicht, warum auch ,,unitary" E.P.S.P.s nach heterosynaptischem Reiz vergrößert auftreten, siehe KANDEL u. TAUC $[16,17]$ und diese Arbeit Abb.8. Das spiegelbild- 
liche Verhalten bei der Amplitudenabnahme des Testpotentials unter Wahrung der Konfiguration vor Erreichen des steady state während wiederholter Testreizung (Abb.7), verglichen mit der Amplituden. zunahme bei H.S.F., spricht weiter dafür, daß es sich in beiden Fällen um eine Veränderung der Transmitterausschüttung an der Einzelsynapse handelt. Bekanntlich steht die Amplitude eines einzelnen synaptischen Potentials in direkter Abhängigkeit von der Menge der Transmitterausschüttung (Ecctes [9]).

Posttetanische Potenzierung würde zwar die vermehrte Transmitterausschüttung erklären, ist aber für diejenigen Testunits unwahrscheinlich, die auch durch lange fortgesetzte Primingreizung nicht erschöpft werden, und daher wahrscheinlich gar nicht entladen worden sind (Abb.4 und 5). Dieselben Testunits werden aber, wenn auch nicht entladen und erschöpft, so dooh durch den Primingreiz in dramatischer Weise dahingehend verändert, daß eine nachfolgende gepaarte Reizung eine besonders ausgiebige, dafür aber kurzlebige H.S.F. ergibt. Eine solche latente Beeinflussung würde am ehesten der oben erwähnten dritten Möglichkeit, nämlich der lokalen heterosynaptischen Kontrolle der Transmitterbereitstellung, entsprechen. Es liegt nahe, hier an axo-axonale präsynaptische Endknopfverbindungen zwischen Priming- und Testendformation zu denken, in denen ein Transmitter der Primingneurone oder eine lokale Hyperpolarisation die Transmitterbereitstellung innerhalb der Testendformation kontrolliert.

Solange noch keine intracellulären Ableitungen der Testneurone vorliegen, läßt sich nicht beurteilen, ob während der H.S.F. eine Hyperpolarisation ihrer Endigungen vorliegt. TAKEUCH $u$. TAKEUCH [22] haben an der Riesensynapse im StellatGanglion des Krebses gefunden, daß Hyperpolarisation der präsynaptischen Seite zu stark gesteigerter Transmitterausschüttung führt. Nach den Ableitungen von HUBBARD u. WrLLIS $[10,11]$ an der neuromuskulären Endplatte im Rattenzwerehfell, kann dieser Effekt einen verzögerten Zeitverlauf zeigen, der in gewisser Hinsicht der H.S.F. ähnelt. Präsynaptische Endknopfverbindungen sind bei Aplysia elektronenoptisch noch nicht gesichert, doch sprechen einige elektrophysiologische Befunde $[23,25,26]$ für das Vorkommen präsynaptischer Hemmung und Förderung im Sinne von Ecoues. Beobachtungen von DEL CASTIL. u. KATZ [5] und von DUDBL u. KUFFLER [7] an einem anderen Beispiel, nämlich an der neuromuskulären Endplatte, zeigen, daB selbst dann, wenn ein Impuls nicht zur Transmitterausschüttung führt, er doch die Transmitterausschüttung für einen nachfolgenden Impuls potenzieren kann.

Das hypothetische Schema der H.S.F. in Abb.10b ist geeignet, die Kurven der Abb. 4 und 5 im Sinne der erwähnten Transmitterakkumulation zu interpretieren. Im Laufe einer fortgesetzten Primingreizung mit nur gelegentlichen ungepaarten Testreizen wird die Testunit 1 durch ihre direkten fördernden Verbindungen mit der Primingseite jedesmal mitentladen, was praktisch dem Ergebnis einer ,gepaarten Reizung" an- 
nähernd gleichkommt. Wahrscheinlich gibt es auch Axonkollateralen der Testneurone, welche in den Primingnerven gehen, und von dort her antidrom entladen werden können. Die Endformation der Testunits wird weiterhin präsynaptisch im Sinne einer vermehrten Transmitterbereit. stellung beeinflußt. Diese vermehrt bereitgestellte Transmittermenge wird durch die in zeitlicher Nachbarschaft eintretenden Aktionspotentiale voll aktiviert und ausgeschüttet, was zu einem besonders großen E.P.S.P. (= H.S.F.) führt. Bei lange fortgesetzter Primingreizung kann das Testneuron den ständigen starken Transmitterverlust nicht mehr durch Synthese kompensieren, wonach Erschöpfung eintritt. Während dieses ganzen Vorganges ist aber im Testneuron 2, welches keine direkt fördernde, sondern nur die präsynaptische Verbindung von der Primingseite her haben soll, Transmitter mobilisiert und akkumuliert worden. Wird jetzt gepaarte Reizung von außen angewandt, so kann auch diese Transmittermenge aktiviert und ausgeschüttet werden. Dieses würde gut die erneut auftretende, besonders große H.S.F. in Abb.5 erklären. Die gemessene Kurzlebigkeit dieser Förderung wäre auch zu erwarten, da nach solcher prolongierter Primingreizung alle verfügbaren Transmitter bereitgestellt und auf einmal ausgeschüttet würden, wonach die Reserven erschöpft wären. Es ist durchaus möglich, daß ein Teil der ,Erschöpfung“ nicht nur auf Transmitterverarmung des präsynaptischen Neurons, sondern außerdem auf Desentisierung [27] der Receptoren an der postsynaptischen Membran zurückgeht.

Falls wirklich präsynaptische Vorgänge eine Rolle bei der H.S.F. spielen sollten, so ist zumindestens noch eine Besonderheit mit zu berücksichtigen: Bei Entladung des Primingneurons tritt zunächst nur die erwähnte Mobilisierung des Transmitters in der Test-Endformation ein. Um diesen Transmitter vollends zu aktivieren, muß ein Aktionspotential in der Membran des Testneurons in zeitticher Nachbarschaft zu dem des Primingneurons erfolgen. Ein zeitlich vom Primingreiz unabhängiges Aktionspotential hat (wie die zeitlich unabhängigen Testreizungen nach Erschöpfung in der Kurve der Abb.4 zeigen) an Testneuronen, die nicht durch Kollateralen der Primingseite entladen werden, nicht denselben Effekt, sondern schüttet nur die normale Menge von Transmitter aus. Erst nachfolgende gepaarte Reizung mit ihrer Aktivierungswirkung führt zur H.S.F.

Die Tatsache, daß Priming alleine in gewissem Umfange zur H.S.F. an mehreren Testnerven führen kann $[16,17]$, wirft die Frage nach der Spezifität der H.S.F., und hinsichtlich der Lernprozesse nach der Abgrenzung von einfacher Sensitivierung auf. Der speziellen Untersuchung dieser Probleme widmet sich eine nachfolgende Arbeit [13].

Es ist zu wünschen, daß elektronenoptische Studien von AplysiaGanglien einen morphologischen Anhalt über die Art der Neuronenverbindungen, welche für die H.S.F. in Frage kommen, liefern mögen. Neurophysiologisch ist zu hoffen, daß es gelingt, Testneurone während der H.S.F. zu identifizieren und intracellulär abzuleiten. Neuropharma- 
kologisch wäre es interessant, die für die H.S.F. verantwortlichen Transmittersubstanzen näher zu identifizieren. Versuche in diesen Richtungen werden gegenwärtig in unserem Labor vorgenommen.

Dr. E. R. KANDEL sei für Beratung bei der Abfassung des Manuskriptes gedankt. Dr. N. Chatazonitis, Dr. L. Tauo und Dr. F. Strumwasser sei für ihre Großzügigkeit gedankt, mit der sie uns bei der initiellen Einrichtung unseres Aplysia-Labors beraten haben. Wir danken auch Fräulein BRIGITTE DöHRMANN für ihre sorgfältige Assistenz bei der Durchführung und Auswertung der Versuche.

\section{Literatur}

1. Arvanttakr, A., et H. Cardot: Observations sur la constitution des ganglions et conducteurs nerveux et sur l'isolement du soma neuronique vivant chez les mollusques gastéropodes. Bull. Histol. Techn. micr. 18, 133-144 (1941).

2. - , et N. Chalazonitis: Activations du soma géant d'aplysia. Par voie antidrome et par voie orthodrome. (Dérivation endocytaire) Extrait des Arch. Sci. physiol. X, Nr. 1, 95-128 (1956).

3. Badmgarten, R. v., and B. Jahan-PaRvaR: Time course of repetitive heterosynaptic facilitation in Aplysia californica. Brain Res. (in press) (1967).

4. Bullock, T. H., and G. A. HoRRIDGE: Structure and function in the nervous systems of invertebrates, pp. 1284-1386. San Francisco and London: Freeman 1965.

5. CASTILlo, J. DEL, L. EngBaEK, and B. Katz: Statistical factors involved in neuromuscular facilitation and depression. J. Physiol. (Lond.) 124, 574-585 $(1954 \mathrm{c})[87,94]$.

6. Doty, R. W., and C. GruRgeA: Conditioned reflexes established by coupling electrical excitation of two cortical areas. In: CIOMS Symposium on Brain mechanism and learning, ed. by J. F. Derafressayer, A. Fessard, and J. Konorsky. Oxford: Blackwell Scientific Publications 1961.

7. Dudel, J., R. Gryder, A. KaJ, S. W. Kuffler, and D. D. Potter: Mechanism of facilitation at the crayfish neuromuscular junction. J. Physiol. (Lond.) 155, 530-542 (1961 b).

8. Ecouts, J. C.: The effects of use and disuse on synaptic function. In Brain mechanismus and learning 334-352, CIOMS symposium. Springfield, Ill.: Ch. C. Thomas 1961.

9. - The physiology of synapses, p. 98. Berlin, Göttingen, Heidelberg: Springer 1964.

10. HUBBARD, J. I.: Repetitive stimulation at the mammalian neuromuscular junction, and the mobilization of transmitter. J. Physiol. (Lond.) 169, $641-662(1963)$.

11. - , and W. D. WILIrS: Mobilization of transmitter by hyperpolarization. Nature (Lond.) 193, 174-175 (1962a).

12. Hugress, J. R.: Post-tetanic potentiation. Physiol. Rev. 38, 91-111 (1958).

13. JaHAN-Parvar, B., u. R. v. Badmgarten: Untersuchungen zur Spezifitätsfrage der heterosynaptischen Facilitation bei Aplysia californica. Pflügers Arch. ges. Physiol. 295, 347-360 (1967).

14. Kandel, E. R., W. T. Frazier, and R. E. Coggeshall: Opposite synaptic actions mediated by different branches of an identifiable interneuron in aplysia. Science 155, 346-349 (1967).

15. KANDEL, E. R., and L. TAUC: Mechanism of prolonged heterosynaptic facilitation. Nature (Lond.) 202, 145-147 (1964). 
16. KANDEL, E. R., L. TAUC, and L.TAUd: Heterosynaptic facilitation in neurons of the abdominal ganglion of Aplysia depilans. J. Physiol. (Lond.) 181, $1-27$ (1965).

17. - - Mechanism of heterosynaptic facilitation in the giant cell of the abdominal ganglion of aplysia depilans. J. Physiol. (Lond.) 181, 28-47 (1965).

18. Llovd, D. P. C.: Post-tetanic potentiation of response in monosynaptic reflex pathways of the spinal cord. J. gen. Physiol. ås, 147-170 (1949).

19. Morret, F.: Electrophysiological contributions to the neural basis of learning. Physiol. Rev. 41, 443-494 (1961).

20. STRUMWASsER, F.: The demonstration and manipulation of a circadian rhythm in a single neuron. In: Circadian clocks, pp. 432-462. Amsterdam: NorthHolland Publishing 1965.

21. - Types of information stored in single neurons. Proceedings of the conference on invertebrate nervous systems (A. G. WIERSMA, ed.) Univ. of Chicago 1966.

22. TAKEUCHI, A., and N. TAKEUCHI: Electrical changes in pre- and postsynaptic axons of the giant synapse of loligo. J. gen. Physiol. 45, 1181-1193 (1962).

23. TAUC, L.: Evidence of synaptic inhibitory actions not conveyed by inhibitory postsynaptic potentials. In: Inhibitions of the nervous system and gamma amino-butyric acid, pp. 85-89. Oxford: Pergammon Press 1960.

24. - Sito of origin and propagation of spike in the giant neuron of aplysia. J. gen. Physiol. 45, 1077-1097 (1962).

25. - Inhibition présynaptique dans les neurones centraux de l'aplysie. C. R. Acad. Sci. (Paris) 259, 885-888 (1964).

26. - Presynaptic inhibition in the abdominal ganglion of Aplysia. J. Physiol. (Lond.) 181, 282-307 (1965).

27. -, and J. BRUNER: "Desensitization" of cholinergic receptors by acetylcholine in molluscan central neurons. Nature (Lond.) 198, 33-34 (1963).

Prof. Dr. R. v. BaUmgarten

Mental Health Research Institute

University of Michigan

Ann Arbor/Michigan, U.S.A. 